\title{
GRAVITOMAGNETIC ULTRARELATIVISTIC INTERACTION
}

\author{
R. M. Plyatsko \\ Pidstryhach Institute for Applied Problems of Mechanics \\ and Mathematics of the National Academy of Sciences of Ukraine, \\ 3-b Naukova Str., Lviv, UA-79601, Ukraine \\ (Received September 16, 1999)
}

\begin{abstract}
The gravitomagnetic components of the gravitational field in the tetrad representation $B_{(k)}^{(i)}$ are considered from the point of view of an observer moving relative to a Schwarzschild source. These components are compared with the gravitoelectric components $E_{(k)}^{(i)}$. The general expressions for $B_{(k)}^{(i)}$ and $E_{(k)}^{(i)}$ are obtained without any restriction on the value of the observer's velocity. The influence of the relativistic Lorentz $\gamma$-factor on the gravitomagnetic and gravitoelectric components is investigated. It is pointed out that in the proper frame of reference of a spinning test particle just the gravitomagnetic interaction determines the deviation of this particle motion from the geodesic motion.

Key words: gravitomagnetic and gravitoelectric fields, ultrarelativistic gravitation, spinning test particle.
\end{abstract}

PACS number(s): 04.20.Cv, 95.30.Sf

\section{INTRODUCTION}

The gravitoelectric and gravitomagnetic interactions as the similarities of the usual electric and magnetic interactions have been considered for a long time [1]. The theoretical investigations have largely been tackled since the experimental tests are difficult because of the small quantities of the corresponding effects. Usually the gravitoelectromagnetic effects were considered for the low velocities and the weak gravitational fields, though on the whole the conception "gravitoelectric (gravitomagnetic) field" was introduced without these restrictions. For example, it is pointed out in Ref. [2] that the gravitomagnetic components of the gravitational field are small provided a source is not moving with a large velocity. It is stressed in Ref. [3] that an analogy between gravitation and electromagnetism exists on the principled level and that the weak field approximation plays a heuristic role only. However, a detailed analysis of this analogy for the large velocities was not carried out.

The investigations of the gravitomagnetic field on the level of the concrete physical effects have a special feature. Namely, as a rule, the gravitomagnetic field appeared due to the momentum of rotation of a mass (mainly it is a source of the Lense-Thirring metric) and not due to its linear momentum (the analogy of the known situation in electrodynamics when the magnetic field is created by a moving electric charge).

In this paper we shall consider the gravitomagnetic interaction in the situation when a Schwarzschild's source of the gravitational field is moving relatively to an observer with the ultrarelativistic velocity.

It is known that in the local orthogonal basis the gravitomagnetic components of the gravitational field $B_{(k)}^{(i)}$ are determined by the relation $[4,5]$

$$
B_{(k)}^{(i)}=-\frac{1}{2} R_{(m)(n)}^{(i)(4)} \varepsilon_{(k)}^{(m)(n)}
$$

where $R^{(i)(4)}(m)(n)$ are the local components of the Riemann tensor, $\varepsilon^{(m)(n)}(k)$ is the Levi-Civita tensor, $\varepsilon^{(1)(2)(3)}=-\varepsilon_{(1)(2)(3)}=1$, signature,,,---+ (here and in the following, the indices of the orthogonal tetrads are placed in the parentheses; Latin indices run 1, 2, 3 and Greek indices do 1, 2, 3, 4). For the gravitoelectric components $E_{(k)}^{(i)}$ we have $[4,5]$

$$
E_{(k)}^{(i)}=R^{(i)(4)}(k)(4)
$$

The relations $B^{(i)(k)}=B^{(k)(i)}, E^{(i)(k)}=E^{(k)(i)}, \operatorname{Sp} B=$ $0, \operatorname{Sp} E=0$ take place.

\section{THE GRAVITOMAGNETIC AND GRAVITOELECTRIC COMPONENTS OF THE GRAVITATIONAL FIELD OF A MOVING SCHWARZSCHILD'S SOURCE}

Let us consider the gravitomagnetic and gravitoelectric components from the point of view of an observer who is moving with any velocity relative to a Schwarzschild's source of the gravitational field. For convenience, we choose the orientation of the spatial coordinate axes of the frame of reference of a moving observer in such a manner: (1) The first axis is perpendicular to the plane that is determined by the direction of motion of an observer and the radial direction to the source (for note of this axis we use the tetrad index (1)); (2) The second axis is directed along the velocity of an observer (this axis we note as (2)). Then according to the results 


\section{R. PLYATSKO}

of Ref. [6] the nonzero components of the tetrads $\lambda_{(\beta)}^{\alpha}$ describing this observer are

$$
\begin{gathered}
\lambda_{(1)}^{2}=\sqrt{-g^{22}}, \quad \lambda_{(2)}^{1}=u^{1} u^{4} \sqrt{\frac{g_{44}}{u_{4} u^{4}-1}}, \\
\lambda_{(2)}^{3}=u^{3} u^{4} \sqrt{\frac{g_{44}}{u_{4} u^{4}-1}}, \quad \lambda_{(2)}^{4}=\sqrt{\frac{u_{4} u^{4}-1}{g_{44}}}, \\
\lambda_{(3)}^{1}=u^{3} \sqrt{\frac{g^{11} g_{33}}{u_{4} u^{4}-1},} \lambda_{(3)}^{3}=-u^{1} \sqrt{\frac{g^{33} g_{11}}{u_{4} u^{4}-1}}, \\
\lambda_{(4)}^{1}=u^{1}, \quad \lambda_{(4)}^{3}=u^{3}, \quad \lambda_{(4)}^{4}=u^{4},
\end{gathered}
$$

where $g_{\mu \nu}$ is the Schwarzschild metric tensor in the standard coordinates $x^{1}=r, \quad x^{2}=\theta, \quad x^{3}=\varphi, \quad x^{4}=t$, $u^{\lambda}$ is the 4 -velocity of an observer $\left(u^{2}=0\right.$ because this observer is moving in the plane $\theta=\pi / 2$ ).

According to (1) for the calculations of the gravitomagnetic components of the gravitational field it is necessary to have the values of the local components of the Riemann tensor $R_{(\alpha)(\beta)(\gamma)(\delta)}$. These components are connected with the global components of this tensor $R_{\mu \nu \rho \sigma}$ by the relation

$$
R_{(\alpha)(\beta)(\gamma)(\delta)}=\lambda_{(\alpha)}^{\mu} \lambda_{(\beta)}^{\nu} \lambda_{(\gamma)}^{\rho} \lambda_{(\delta)}^{\sigma} R_{\mu \nu \rho \sigma}
$$

The nonzero components of the Riemann tensor in the standard Schwarzschild coordinates for $\theta=\pi / 2$ are given by

$$
\begin{gathered}
R_{1212}=R_{1313}=\frac{m}{r-2 m}, \quad R_{2323}=-2 m r \\
R_{1414}=\frac{2 m}{r^{3}}, \quad R_{2424}=R_{3434}=-\frac{m}{r}\left(1-\frac{2 m}{r}\right) .
\end{gathered}
$$

The values of the three local components of the Riemann tensor, namely $R_{(i)(4)(2)(3)}$, are presented in Ref. [6]:

$$
\begin{gathered}
R_{(1)(4)(2)(3)}=0 \\
R_{(2)(4)(2)(3)}=-\frac{3 m u^{1} u^{3}}{r^{2} \sqrt{u_{4} u^{4}-1}}\left(1-\frac{2 m}{r}\right)^{-1 / 2}, \\
R_{(3)(4)(2)(3)}=-\frac{3 m u^{3} u^{3} u^{4}}{r \sqrt{u_{4} u^{4}-1}}\left(1-\frac{2 m}{r}\right)^{1 / 2} .
\end{gathered}
$$

For the calculation of the six other local components of this tensor which according to (1) we need for the calculation of $B_{(k)}^{(i)}$, namely $R_{(1)(4)(1)(2)}, R_{(1)(4)(1)(3)}$, $R_{(2)(4)(1)(2)}, R_{(2)(4)(1)(3)}, R_{(3)(4)(1)(2)}, R_{(3)(4)(1)(3)}$, we insert values $(3)$ and $(5)$ into $(4)$. Then we obtain

$$
\begin{aligned}
& R_{(1)(4)(1)(2)}=-\frac{3 m u^{3} u^{3} u^{4}}{r \sqrt{u_{4} u^{4}-1}}\left(1-\frac{2 m}{r}\right)^{1 / 2}, \\
& R_{(1)(4)(1)(3)}=\frac{3 m u^{1} u^{3}}{r^{2} \sqrt{u_{4} u^{4}-1}}\left(1-\frac{2 m}{r}\right)^{-1 / 2}, \\
& R_{(2)(4)(1)(2)}=0, \quad R_{(2)(4)(1)(3)}=0, \\
& R_{(3)(4)(1)(2)}=0, \quad R_{(3)(4)(1)(3)}=0 .
\end{aligned}
$$

Using (6), (7) in (1) we find such nonzero components of the gravitomagnetic field:

$$
\begin{aligned}
& B_{(2)}^{(1)}=B_{(1)}^{(2)}=\frac{3 m u_{\|} u_{\perp}}{r^{3} \sqrt{u_{4} u^{4}-1}}\left(1-\frac{2 m}{r}\right)^{-1 / 2}, \\
& B_{(3)}^{(1)}=B_{(1)}^{(3)}=\frac{3 m u_{\perp}^{2} u^{4}}{r^{3} \sqrt{u_{4} u^{4}-1}}\left(1-\frac{2 m}{r}\right)^{1 / 2},
\end{aligned}
$$

where $u_{\|}=u^{1}$ is the radial component of the 4-velocity and $u_{\perp}=r u^{3}$ is the tangential component. By the condition $u_{\mu} u^{\mu}=1$ we take into account that in (8)

$$
\sqrt{u_{4} u^{4}-1}=\left[u_{\perp}^{2}+\left(1-\frac{2 m}{r}\right)^{-1} u_{\|}^{2}\right]^{1 / 2}
$$

We stress that relations (8) are valid for any velocity of an observer, including the ultrarelativistic velocity.

It is easy to see that the components of the gravitomagnetic field (8) are nonzero only if $u_{\perp} \neq 0$, i.e. if the motion of an observer is nonradial. A similar property takes place in electrodynamics for the magnetic field of a moving charge. However, it is necessary to remember that on the level of the analogies the tensor $B^{(i)(k)}$ does not correspond to the vector of the magnetic field in electrodynamics but to the first derivatives from this vector with respect to the spatial coordinates (likewise, as $E^{(i)(k)}$ corresponds to the gradient of the vector of the electric field [5]). Therefore, the main dependence of components (8) from the radial coordinate is $1 / r^{3}$ and not $1 / r^{2}$.

The values of components (8) essentially depend on the velocity of an observer: for the low velocity, when $\left|u_{\|}\right| \ll 1,\left|u_{\perp}\right| \ll 1, u^{4} \approx 1$, the factor $m / r^{3}$ in (8) is multiplied by the corresponding small quantities, while for the ultrarelativistic velocity, when $\left|u_{\|}\right| \gg 1,\left|u_{\perp}\right| \gg$ $1, u^{4} \gg 1$, this factor is multiplied by the large quantities. Moreover, since $u_{\|}, u_{\perp}, u^{4}$ are proportional to the relativistic Lorentz $\gamma$-factor, according to (8) the components $B_{(2)}^{(1)}=B_{(1)}^{(2)}$ are proportional to the $\gamma$-factor and the components $B_{(3)}^{(1)}=B_{(1)}^{(3)}$ are proportional to the 
square of the $\gamma$-factor. (It is known that in electrodynamics the components of the vector of the magnetic field for an electric charge moving with the ultrarelativistic velocity are proportional to the Lorentz $\gamma$-factor).

For further analysis of the gravitomagnetic components of the gravitational field let us compare (8) with the gravitoelectric components of this field. For this purpose we shall consider the components $E_{(k)}^{(i)}$ using (2). Inserting (3) into (4) and taking into account (5) after the corresponding calculations we obtain the nonzero components $E_{(k)}^{(i)}$ :

$$
\begin{aligned}
& E_{(1)}^{(1)}=\frac{m}{r^{3}}\left(1+3 u_{\perp}^{2}\right), \\
& E_{(2)}^{(2)}=-\frac{2 m}{r^{3}}+\frac{3 m}{r^{3}} \frac{u_{\perp}^{2}}{u_{4} u^{4}-1}, \\
& E_{(3)}^{(2)}=E_{(2)}^{(3)}=-\frac{3 m}{r^{3}} \frac{u_{\|} u_{\perp} u^{4}}{u_{4} u^{4}-1}, \\
& E_{(3)}^{(3)}=\frac{m}{r^{3}}-\frac{3 m}{r^{3}} \frac{u_{\perp}^{2} u_{4} u^{4}}{u_{4} u^{4}-1} .
\end{aligned}
$$

One can see that for the low velocity all components (10) have the order $m / r^{3}$, i.e. are much larger than the components $B_{(k)}^{(i)}$ from (8) for this velocity. (It accords with the known fact that the tensor of the gravitoelectric field $E_{(k)}^{(i)}$, in contrast with $B_{(k)}^{(i)}$, has the Newtonian limit [5]). While for the ultrarelativistic velocity according to (10)

$$
\begin{gathered}
E_{(1)}^{(1)} \approx \frac{3 m}{r^{3}} \gamma^{2}, \quad E_{(2)}^{(2)} \approx \frac{3 m}{r^{3}}, \\
E_{(3)}^{(2)}=E_{(2)}^{(3)} \sim \frac{3 m}{r^{3}} \gamma, \quad E_{(3)}^{(3)} \sim \frac{3 m}{r^{3}} \gamma^{2} .
\end{gathered}
$$

That is for this velocity the maximum values of the components $B_{(k)}^{(i)}$ in (8) and $E_{(k)}^{(i)}$ in (10) have the same order, namely, $3 \gamma^{2} m / r^{3}$.

Thus, if for the low velocity the gravitoelectric field prevails as compared with the gravitomagnetic field, for the ultrarelativistic velocity both the components of the gravitational field are comparable. For the concrete estimates we shall consider the equations which demonstrate the influence of $B_{(k)}^{(i)}$ and $E_{(k)}^{(i)}$. Concerning $E_{(k)}^{(i)}$ it is known that this tensor is presented in the equation of the geodesic deviation [5]

$$
\frac{D^{2} l^{(i)}}{d s^{2}}=l^{(k)} E_{(k)}^{(i)}
$$

where $s$ is the natural parameter, $l^{(i)}$ is the vector of the deviation of the two close geodesic lines. Just the deviation equation is used in [2] for the analysis of the tidal forces acting on an observer falling into a black hole. According to (12) for the tidal acceleration $a_{\text {tidal }}^{(i)}$ we have

$$
a_{\text {tidal }}^{(i)}=l^{(k)} E_{(k)}^{(i)}
$$

(particularly, in the Newtonian approximation $E_{(k)}^{(i)}$ is the "Newtonian tidal matrix" [5]). However, in Ref. [2], Sec. 31.2 the analysis of the tidal forces was restricted to the radial motion $\left(u_{\perp}=0\right)$ when from (10) we have

$$
\begin{gathered}
E_{(1)}^{(1)}=\frac{m}{r^{3}}, \quad E_{(2)}^{(2)}=-\frac{2 m}{r^{3}}, \\
E_{(3)}^{(2)}=E_{(2)}^{(3)}=0, \quad E_{(3)}^{(3)}=\frac{m}{r^{3}} .
\end{gathered}
$$

Relations (14) are valid for any velocity of the radial motion. Even if $u_{\|}$is ultrarelativistic, the influence of the Lorentz $\gamma$-factor is absent in (14). So, the radial motion is particular in the sense of this case does not discover the special features of the gravitoelectric interaction and the tidal forces just for the ultrarelativistic velocity. If $u_{\perp} \neq 0$ these features are clear from (10), (12). Therefore, the analysis of the tidal forces acting on an observer falling into a black hole from Ref. [2] may be supplemented. The main point, as we see, is the conclusion that the tidal forces essentially grow not only near the horizon surface but far from this surface also if the velocity of the nonradial fall of an observer is ultrarelativistic.

The results of Ref. [6] demonstrate that according to the Mathisson-Papapetrou equations in the comoving frame of reference just the gravitomagnetic components of the gravitational field act on the spin of a test particle. This action has the form of the gravitational spinorbit interaction. If for the observer comoving with the spinning test particle in a Schwarzschild field the tetrad components are chosen as we described on the beginning of this Section and if the spin is directed along the spatial direction with the tetrad index (1), then according to $[6]$ and $(7),(8)$ for the 3 -vector of the spin-orbit acceleration $a_{(i)}$ we have

$$
a_{(i)}=\frac{s_{(1)}}{M} B_{(i)}^{(1)}
$$

where $s_{(1)}=$ const is the only nonzero component of the spin 4 -vector, $M$ is the mass of the spinning test particle. (According to (8) the nonzero components in (15) are $B_{(2)}^{(1)}$ and $\left.B_{(3)}^{(1)}\right)$. Though expressions (13), (15) are similar their meaning is different. If acceleration (13) is the measure of the relative deviation of the two close geodesic lines, then acceleration (15) is the measure of the nongeodesic properties of the one world line, namely, the world line of the spinning test particle [6]. It being known that this nongeodesity grows as $\gamma^{2}$ correspondingly as the components $B_{(k)}^{(i)}$ in (8) depend on the Lorentz $\gamma$-factor. Though in the ultrarelativistic region, as ws stressed above, the value of acceleration (13) grows correspondingly also, the nature of the forces that cause 
accelerations (13) and (15) is different. According to the analogy with electromagnetism one can say that acceleration (13) is similar to the acceleration that is caused by the difference of the values of the electric field in the two close spatial points while acceleration (15) is similar to the usual spin-orbit interaction [7]. Let alone this fact that acceleration (13) does not disappear in the Newtonian limit while (15) is the relativistic effect.

The gravitomagnetic components in the MathissonPapapetrou equations are distinguished in the obvious form in [8]. However, the corresponding analysis of the influence of these components on the behaviour of a spinning test particle just for the ultrarelativistic velocity was not carryed out in [8]. From the other side, though in [5] one can see the relativistic Lorentz $\gamma$-factor in the expressions for the gravitoelectric and gravitomagnetic components of the gravitational field, in the context of the Mathisson-Papapetrou equations these expressions were not considered .

Obviously, if we pass from the concrete frame of reference to other one, the gravitoelectric and gravitomagnetic components are mixed according to the relations describing this passing. However, if the frame of reference is fixed (in our case it is the comoving frame of reference), then the components $B_{(k)}^{(i)}$ differ from $E_{(k)}^{(i)}$ according to (1), (2).

Since in the frame of reference comoving with the spinning test particle just the gravitomagnetic components of the gravitational field determine the nongeodesic motion of this particle, let us consider these components in an interesting case of motion.

\section{THE GRAVITOMAGNETIC FIELD IN THE FRAME OF REFERENCE COMOVING WITH THE SPINNING TEST PARTICLE ON THE ULTRARELATIVISTIC CIRCULAR ORBITS SUSPENDING OVER A SCHWARZSCHILD'S SOURCE}

It is known $[9,10]$ that according to the MathissonPapapetrou equations the important consequence of the gravitational ultrarelativistic spin-orbit interaction is the existence of the essentially nongeodesic circular orbits of a spinning test particle in a Schwarzschild field. Here we shall consider the components of the gravitomagnetic field from the point of view of the observer comoving with the particle on these orbits. The nonzero components of the comoving tetrads are [11]

$$
\begin{aligned}
& \lambda_{(1)}^{1}=-g^{11}\left(-g^{22}\right)^{-1 / 2} \frac{S_{r}}{S_{\theta}}\left[1+\frac{g^{11}}{g^{22}}\left(\frac{S_{r}}{S_{\theta}}\right)^{2}\right]^{-1 / 2} \\
& \lambda_{(1)}^{2}=\left(-g^{22}\right)^{-1 / 2}\left[1+\frac{g^{11}}{g^{22}}\left(\frac{S_{r}}{S_{\theta}}\right)^{2}\right]^{-1 / 2}, \\
& \lambda_{(2)}^{3}=u^{3} u^{4}\left(\frac{g_{44}}{u_{4} u^{4}-1}\right)^{1 / 2}
\end{aligned}
$$

$$
\begin{aligned}
& \lambda_{(2)}^{4}=\left(\frac{u_{4} u^{4}-1}{g_{44}}\right)^{1 / 2}, \\
& \lambda_{(3)}^{1}=u^{3}\left(\frac{g^{11} g_{33}}{u_{4} u^{4}-1}\right)^{1 / 2}\left[1+\frac{g^{11}}{g^{22}}\left(\frac{S_{r}}{S_{\theta}}\right)^{2}\right]^{-1 / 2}, \\
& \lambda_{(3)}^{2}=-\frac{S_{r}}{S_{\theta}} \lambda_{(3)}^{1}, \quad \lambda_{(4)}^{3}=u^{3}, \quad \lambda_{(4)}^{4}=u^{4},
\end{aligned}
$$

where $S_{r}, S_{\theta}$ are the components of the spin 3-vector connected by the relation

$$
S_{r}\left(1-\frac{3 m}{r}\right)+S_{\theta} \frac{\cos \theta}{r \sin \theta}=0 .
$$

Spatial direction (1) in (16) is connected with the spin orientation and direction (2) coincides with the direction of the particle's motion on the circular orbit. For the calculation of the gravitomagnetic components $B_{(k)}^{(i)}$ we shall use general relations (1), (4) and the generalization of expressions (5) for any values of the angle $\theta$ :

$$
\begin{aligned}
& R_{1212}=\frac{m}{r-2 m} \\
& R_{1313}=\frac{m}{r-2 m} \sin ^{2} \theta \\
& R_{2323}=-2 m r \sin ^{2} \theta \\
& R_{4141}=\frac{2 m}{r^{3}} \\
& R_{4242}=-\frac{m}{r}\left(1-\frac{2 m}{r}\right) \\
& R_{4343}=-\frac{m}{r}\left(1-\frac{2 m}{r}\right) \sin ^{2} \theta
\end{aligned}
$$

Inserting (17) into (4) and using this result in (1) we find the nonzero components of $B_{(k)}^{(i)}$ :

$$
\begin{aligned}
B_{(1)}^{(1)}= & -B_{(3)}^{(3)}=-R_{(1)(4)(2)(3)}=\frac{m}{r^{3}} u_{\perp} u^{4} \cos \theta, \\
B_{(3)}^{(1)}= & B_{(1)}^{(3)}=-R_{(3)(4)(2)(3)}= \\
& -\frac{m}{r^{3}} u_{\perp} u^{4}\left(4-\frac{9 m}{r}\right)\left(1-\frac{2 m}{r}\right)^{-1 / 2} \sin \theta .
\end{aligned}
$$

Because the velocity of the spinning test particle on the suspending circular orbits in a Schwarzschild field is ultrarelativistic $[9,10]$, all components (19) are proportional to the square of the relativistic Lorentz $\gamma$-factor. So, from the point of view of the observer comoving with the particle the gravitomagnetic components (19) 
have the large values. This fact demonstrate the essential difference of the suspending ultrarelativistic circular orbits of the spinning test particle in a Schwarzschild field from the so-called Weyssenhoff orbits in the Minkowski spacetime [12]. In the first case we see the physical reason changing the trajectory of the spinning particle as compared with the corresponding trajectory of the nonspinning particle, namely, this reason is the gravitational spin-orbit interaction. From the point of view of the comoving observer this interaction is determined just by gravitomagnetic components (19). Indeed, according to Ref. [11] and (19) the nongeodesic spin-orbit acceleration $a_{(i)}$ of the particle is determined by the expressions

$$
\begin{aligned}
& a_{(1)}=\frac{s_{(1)}}{M} B_{(1)}^{(1)}, \\
& a_{(2)}=0 \\
& a_{(3)}=\frac{1}{2} \frac{s_{(1)}}{M} B_{(3)}^{(1)} .
\end{aligned}
$$

While in the second case the Weyssenhoff circular orbits in the Minkowski spacetime have the kinematic nature connected with the properties of the center of mass in the relativistic mechanics [13] and the existence of these orbits is not connected with any interaction.

We point out that Eq. (20) is similar to Eq. (15) with the difference that Eq. (15) concerns any equatorial motion while Eq. (20) is deduced for the circular nonequatorial orbits. Besides that Eq. (15) is written accurate to the linear spin terms while Eq. (20) is valid when all the spin terms are taken into account.

\section{CONCLUSION}

The statement that the gravitomagnetic components of a gravitational field are small provided the source of this field is not moving with the large velocity is presented in Ref. [2], Sec. 1.6. The above calculations develop this statement and let us estimate quantatively the influence of the relativistic Lorentz $\gamma$-factor on the values of the gravitomagnetic components. It is important that relations (8), (15) indicate obviously the object which responds directly to the gravitomagnetic field, namely, the spinning test particle (for any value of its velocity relative to the source of the Schwarzschild field). We stress that in [2] the deviation of the spinning test particle from the geodesic trajectory is not connected with the action of the gravitomagnetic field existing in the frame of reference comoving with this particle. On the contrary, in Ref. [2], exercise 40.8 the reason for this deviation is named the tidal forces, and in [2], Sec. 1.6 these forces are considered only as connected with the gravitoelectric components of a gravitational field.

Relations (10) determine the dependence of the gravitoelectric components on the source velocity, and together (8) and (10) can be considered as the similarities of the known electrodynamical relations describing the electromagnetic field of a moving electric charge. Comparing (8) and (10) let us estimate the difference between the gravitomagnetic and gravitoelectric components of a gravitational field for any velocity of its Schwarzschild source. If for the low velocity the gravitomagnetic components are much less than the gravitoelectric components, then for the ultrarelativistic velocity they become comparable. The corresponding relations are valid for the physical effects caused by these components. For example, for a model particle with the mass and spin that are equal to the mass and spin of an electron and with the linear size about $10^{-13} \mathrm{~cm}$ the ratio $\left|\mathbf{a}_{\text {spin-orb }}\right| /\left|\mathbf{a}_{\text {tidal }}\right|$ according to $(13)$, (15) is equal to $\approx 10^{3}$ for the ultrarelativistic velocity while for the low velocity this ratio is much less then 1. (For the calculation of this ratio it is necessary to insert into (13) and (15) the numerical values of the corresponding quantities in an units where $G=c=1$ because all the relations above are written in these units).

The other direct example demonstrating the increase of the influence of the gravitomagnetic field on a spinning test particle in the ultrarelativistic case is the suspending circular orbits in a Schwarzschild field from Sec. III. Further studies will discover the influence of the gravitomagnetic ultrarelativistic interaction on the behaviour of a real quantum particle with a spin in a gravitational field.
[1] A. Matte, Can. J. Math. 5, 1 (1953); W. Campbell, T. Morgan, Am. J. Phys. 44, 356 (1975); K. Nordtvedt, Int. J. Theor. Phys. 27, 1395 (1988); C. Massa, Ann. Phys. (DDR) 46, 159 (1989); B. Mashhoon, H.-J. Paik, C. Will, Phys. Rev. D 39, 2825 (1989); S. Casotto, I. Ciufolini, F. Vespe, G. Bianco, Nuovo Cimento B 105, 589 (1990); R. Jantzen, P. Carini, Ann. Phys. (N.Y.) 215, 47 (1992); B. Mashhoon, Phys. Lett. A 173, 347 (1993); D. Bini, P. Carini, R. Jantzen, D. Wilkins, Phys. Rev. D 49, 2820 (1994); C. McIntosh, R. Arianrhod, S. Wade, C. Hoenselaers, Gen. Relativ. Gravit. 11, 1555 (1994); C. Sopuerta, R. Maartens, G. Ellis, W. Lesame, Phys.
Rev. D 60, 024006 (1999); M. Noun-Zonoz, Phys. Rev. D 60, 024013 (1999).

[2] C. W. Misner, K. S. Thorne, J. A. Wheeler, Gravitation (Freeman, San Francisco, 1973).

[3] N. V. Mitskevich, Fizichieskiie Polia v Obshchei Teorii Otnositiel'nosti (Physical Fields in General Relativity) (Nauka, Moscow, 1969).

[4] K. Thorne, J. Hartle, Phys. Rev. D 31, 1815 (1985).

[5] O. B. Karpov, preprint MIFI 029-89, Moscow, (1989).

[6] R. Plyatsko, Phys. Rev. D 58, 084031 (1998).

[7] R. Wald, Phys. Rev. D 6, 406 (1972).

[8] O. B. Karpov, preprint MIFI 012-90, Moscow, (1990). 


\title{
R. PLYATSKO
}

[9] R. Plyatsko, A. Vynar, Sov. Phys. Dokl. 27, 328 (1982).

[10] R. M. Plyatsko, Proiavy Gravitatsiinoi Ul'trareliatyvists 'koi Spin-Orbital'noi Vzajemodii (Manifestations of Gravitational Ultrarelativistic Spin-Orbit Interaction) (Naukova Dumka, Kyiv, 1988).
[11] R. Plyatsko, S. Pukas, Ukr. Fiz. Zh. 40, 517 (1995).

[12] J. Weyssenhoff, A. Raabe, Acta Phys. Pol. 9, 7 (1947).

[13] C. Möller, The Theory of Relativity (Oxford University Press, Oxford, 1972).

\section{ГРАВІТОМАГНЕТНА УЛЬТРАРЕЛЯТИВІСТИЧНА ВЗАЕМОДІЯ}

\author{
Р. М. Пляाко \\ Інститут прикладних проблем механіки і математики ім. Я. С. Підстригача НАН Украӥни, \\ вул. Наукова, 3-6, Львів, 79601, e-mail:plyatsko@lms.lviv.ua
}

Досліджено гравітомагнетні компоненти гравітаційного поля в тетрадному представленні $B_{(k)}^{(i)}$ з точки зору спостерігача, який рухається відносно шварцшільдівського джерела, і проведено іх зіставлення з гравітоелектричними компонентами $E_{(k)}^{(i)}$. Одержано загальні вирази цих компонент без обмежень на величину швидкости спостерігача. З'ясовано вплив релятивістського $\gamma$-фактора Лоренца на гравітомагнетні та гравітоелектричні компоненти в ультрарелятивістичній області пвидкости. Установлено, що в щій області максимальні значення $B_{(k)}^{(i)}$ і $E_{(k)}^{(i)}$ е однопорядковими, тоді як при невеликій швидкості компоненти $E_{(k)}^{(i)}$ домінують над $B_{(k)}^{(i)}$. Підкреслено, що у власній системі відліку пробної частинки зі спіном саме гравітомагнетна взаємодія визначає відхилення їі руху від геодезійного. 